\title{
A Corynebacterium glutamicum mutant with a defined deletion within the rp/K gene is impaired in (p)ppGpp accumulation upon amino acid starvation
}

\author{
Lutz Wehmeier, ${ }^{1}$ Olaf Brockmann-Gretza, ${ }^{1}$ Agustin Pisabarro, ${ }^{2}$ \\ Andreas Tauch, ${ }^{1}$ Alfred Pühler, ${ }^{1}$ Juan F. Martin ${ }^{2}$ and Jörn Kalinowski ${ }^{1}$
}

Author for correspondence: Jörn Kalinowski. Tel: +49521 106 4825. Fax: +495211065626. e-mail: joern.kalinowski@genetik.uni-bielefeld.de

1 Lehrstuhl für Genetik, Fakultät für Biologie, Universität Bielefeld, D-33501 Bielefeld, Germany

2 Area Microbiologia, Dpto Ecologia, Genetica y Microbiologia, Facultad de Biologia, Universidad de Leon, Campus de Vegazana, E-24071 Leon, Spain

\begin{abstract}
The rp/K gene of Corynebacterium glutamicum ATCC13032 comprises 438 nucleotides and encodes a protein of 145 amino acids with a molecular mass of 15.3 $\mathrm{kDa}$. The amino acid sequence revealed extensive similarities to the large ribosomal subunit protein L11 from several Gram-positive and Gram-negative bacteria. The $C$. glutamicum rp/K gene is located downstream of secE, representing part of the protein export apparatus, and of nusG, encoding a transcription antiterminator protein. The rp/K gene is followed by an ORF homologous to rpIA encoding the 505 ribosomal protein L1. Northern analysis revealed that transcription of the $r p / K-r p / A$ cluster resulted in two different transcripts of 1.5 and $0.6 \mathrm{~kb}$. The $1.5 \mathrm{~kb}$ transcript corresponds to the entire rp/K-rp/A cluster and the short transcript originates from the rp/K gene. A C. glutamicum rp/K mutant strain carrying a 12 bp in-frame deletion within rp/K, which resulted in the loss of the tetrapeptide Pro-Ala-Leu-Gly in the L11 protein, was constructed. The mutant failed to accumulate (p)ppGpp in response to amino acid starvation and exhibited an increased tolerance to the antibiotic thiostrepton. Evidently, the $C$. glutamicum rp/K gene is required for (p)ppGpp accumulation upon nutritional starvation.
\end{abstract}

Keywords: stringent response, (p)ppGpp, Corynebacterium, relA, spoT

\section{INTRODUCTION}

Bacteria have a common adaptational response to amino acid depletion, termed the stringent response, which is mediated by the hyperphosphorylated guanosine nucleotides (p)ppGpp. The accumulation of (p)ppGpp also occurs in response to a variety of other environmental or nutrient stresses such as heat shock, cold shock and carbon source or phosphate limitation. An elevated (p)ppGpp level leads to a complex modulation of gene expression, including the restriction of stable RNA synthesis, the stimulation of certain anabolic genes, and the induction of stationary-phase-specific genes (reviewed by Cashel et al., 1996).

There are two different sources of (p)ppGpp accumulation in Escherichia coli, depending on the nature of the

The GenBank accession number for the $5.25 \mathrm{~kb}$ rp/K region reported in this paper is AF130462. nutritional stress imposed. The RelA protein, responsible for the (p)ppGpp biosynthesis during amino acid deprivation, is a ribosome-associated enzyme catalysing the transfer of a pyrophosphoryl group from ATP to the 3'-hydroxyl position of GTP or GDP. The enzyme is active when uncharged codon-specific tRNAs bind to the ribosomal A-site (Cochran \& Byrne, 1974; Goldman \& Jakubowski, 1990). Furthermore, a functional $50 \mathrm{~S}$ ribosomal protein L11, encoded by the $r p l K$ gene, is required for the activation of the RelA protein and thus for the synthesis of (p)ppGpp (Friesen et al., 1974). However, relA mutant strains continue to accumulate (p)ppGpp in a spoT gene-dependent manner during energy-source exhaustion (Ryals et al., 1982; Murray \& Bremer, 1996). The absence of all detectable (p)ppGpp in relA/spoT double mutants (Xiao et al., 1991) and the fact that RelA and SpoT have extensive amino acid sequence similarity throughout their length (Metzger et al., 1989) led to the proposal that SpoT is a bifunctional enzyme, responsible for (p)ppGpp degradation and for 
the second source of (p)ppGpp-forming activity in E. coli (Murray \& Bremer, 1996).

In contrast to the situation described for E. coli, data from genome sequencing projects of Mycobacterium tuberculosis (Cole et al., 1998), Bacillus subtilis (Kunst et al., 1997), and Mycoplasma genitalium (Fraser et al., 1995) revealed the presence of only one relA/spoT homologue in each of these bacteria. Moreover, the relA/spoT homologous genes from Streptococcus equisimilis H46A and Streptomyces coelicolor A3(2) are bifunctional, encoding a (p)ppGpp synthetase and a (p)ppGpp degrading activity (Mechold et al., 1996; Martinez-Costa et al., 1998). These findings supported the idea that in some bacteria only one gene might substitute for both functions encoded by the relA and spoT genes of E. coli. However, in B. subtilis and $S$. coelicolor A3(2) it has been shown that, like in E. coli, a functional $r p l K$ gene product is required for (p)ppGpp accumulation upon amino acid starvation (Smith et al., 1980; Ochi et al., 1997).

The Gram-positive soil bacterium Corynebacterium glutamicum is the most important micro-organism involved in the industrial production of amino acids such as L-glutamate, L-lysine and L-isoleucine (Malumbres \& Martin, 1996). In the past decade, molecular genetic techniques have been developed for this micro-organism and numerous genes involved in amino acid biosynthesis have been cloned and characterized. Since then, the industrial production of selected amino acids has been improved by genetic engineering and amplification of relevant structural genes. More recently, a relA/spoT homologous gene from C. glutamicum, termed rel, has been reported and shown to encode a bifunctional enzyme with (p)ppGpp synthetase and (p)ppGpp degrading activities (Wehmeier et al., 1998). Moreover, RNA synthesis and growth rate of Brevibacterium flavum are inversely correlated with the cellular ppGpp concentration (Ruklisha et al., 1995), supporting an essential role of the rel gene in the stringent response of this bacterium.

In the present study, we continue our work on the (p)ppGpp metabolism of C. glutamicum with the identification and molecular characterization of the rplK gene showing significant similarity to ribosomal largesubunit proteins L11. (p)ppGpp accumulation assays in wild-type and in $r p l K$ mutant strains strongly indicates that the $r p l K$ gene is indispensable for (p)ppGpp accumulation following amino acid deprivation in $C$. glutamicum.

\section{METHODS}

Bacterial strains, plasmids and growth conditions. All bacterial strains and plasmids used in this study are listed in Table 1. E. coli and C. glutamicum strains were routinely grown in Luria-Bertani (LB) medium (Sambrook et al., 1989) at $37^{\circ} \mathrm{C}$ and $30^{\circ} \mathrm{C}$, respectively. Antibiotics used for plasmid selection were kanamycin $\left(50 \mu \mathrm{g} \mathrm{ml}^{-1}\right.$ for E. coli; $25 \mu \mathrm{g} \mathrm{ml}^{-1}$ for C. glutamicum $)$ and chloramphenicol $\left(50 \mu \mathrm{g} \mathrm{m}^{-1}\right.$ for E. coli; $10 \mu \mathrm{g} \mathrm{ml}^{-1}$ for C. glutamicum).
DNA manipulation and DNA transfer. Plasmid DNA from E. coli was extracted by the alkaline lysis technique (Sambrook et al., 1989). Chromosomal DNA of C. glutamicum was isolated as described previously (Tauch et al., 1995). DNA restriction, agarose gel electrophoresis, Klenow treatment and ligation were carried out as described by Sambrook et al. (1989). Enzymes for DNA manipulation were purchased from Pharmacia or from Boehringer. DNA restriction fragments required for cloning were isolated from agarose gels using the US BiocleanMP Kit (United States Biochemicals). E. coli DH $5 \propto$ MCR (Grant et al., 1990) was used for all cloning procedures. Plasmid DNA was introduced into E. coli strains by electroporation (Tauch et al., 1994). Intergeneric conjugal transfer of plasmids from E. coli to C. glutamicum followed the mating procedure described by Schäfer et al. (1990). Transconjugants were selected on LB agar containing $50 \mu \mathrm{g}$ nalidixic acid $\mathrm{ml}^{-1}$ and $25 \mu \mathrm{g}$ kanamycin $\mathrm{ml}^{-1}$.

Polymerase chain reactions. The synthetic oligonucleotides P1-up (5'-AGGAGCAGGCTGTTGTCACC-3'), P1-down (5'-extension-CGCCGTGAGC-5'-GCCAACTGGAGGAGCAGGGT-3'), and P2-up (5'-extension-TCCAGTTGGC-5'GCTCACGGCGTCAACATCAG-3'), P2-down (5'-GCGGATAGCTACGTCGATGG-3') were used for the amplification of two $600 \mathrm{bp}$ fragments comprising the $5^{\prime}$ and $3^{\prime}$ regions of the C. glutamicum rplK gene, respectively, with $P f u$ DNA polymerase (Stratagene). The construction of the chromosomal $12 \mathrm{bp}$ deletion within the rplK gene was analysed with the primers Pdel-up (5'-TGGCCCAGCACTTGGT- $\left.3^{\prime}\right)$ and Pdel-down (5'-GCAATAGCAACGTCGA$\left.3^{\prime}\right)$. The oligonucleotides P1-up and P2-down were used to amplify a fragment comprising the $r p l K$ wild-type gene. PCR experiments were carried out with a PCT-100 thermocycler (MJ Research). Initial denaturation was conducted at $94{ }^{\circ} \mathrm{C}$ for $2 \mathrm{~min}$ followed by $90 \mathrm{~s}$ denaturation, $90 \mathrm{~s}$ annealing at $58^{\circ} \mathrm{C}$ and extension at $72{ }^{\circ} \mathrm{C}$ for $2 \mathrm{~min}$. This cycle was repeated 30 times followed by a final extension step for $10 \mathrm{~min}$ at $72{ }^{\circ} \mathrm{C}$. PCR products were purified using the PCR Purification Spin Kit (Qiagen).

Southern hybridization. Chromosomal DNA of C. glutamicum was digested with restriction enzymes, separated on $0.8 \%$ agarose gels, and blotted onto Hybond-N nylon membranes (Amersham) using the vacuum blotter Vacu-Gene (Pharmacia). Fixation of DNA, labelling of DNA probes and hybridization were performed with the non-radioactive DIG DNA Labelling and Detection Kit from Boehringer.

DNA sequencing and sequence analysis. During analysis of transposon insertions in C. glutamicum, the Tn5516invers insertion of the transconjugant C. glutamicum KM6 was cloned by applying the plasmid rescue technique (Tauch, 1996). For this purpose, total DNA of C. glutamicum KM6 was isolated, cut with SalI and religated. The ligation mixture was subsequently transformed into E. coli DH5 $\alpha$ MCR. The resulting plasmid pKM6-6, carrying a $5.8 \mathrm{~kb}$ contiguous DNA fragment of the C. glutamicum chromosome, was used as a template for nucleotide sequencing by primer walking, performing the recommended procedures of the Prism Ready Reaction Dye Deoxy Termination Kit (Applied Biosystems) in conjunction with a Prism 377 DNA sequencer (Applied Biosystems). Computer-assisted compilation of the DNA sequences was conducted with the Staden software package (Staden, 1986). Searches for amino acid similarities were carried out with the BLAST programs (Altschul et al., 1997). Global alignments between protein sequences were calculated with the ALIGN computer program (Myers \& Miller, 1988). The prediction of protein secondary structures was calculated 
The C. glutamicum rplK gene region

Table 1. Bacterial strains and plasmids used in this work

\begin{tabular}{|c|c|c|}
\hline $\begin{array}{l}\text { Strain or } \\
\text { plasmid }\end{array}$ & Relevant characteristic* & Source or reference \\
\hline \multicolumn{3}{|c|}{ C. glutamicum strains } \\
\hline RES167 & Restriction-deficient derivative of ATCC13032, $\Delta(\operatorname{cglIR} \operatorname{cglIIR}), \mathrm{Nx}^{\mathrm{R}}$ & $\begin{array}{l}\text { Strain Collection, Lehrstuhl für } \\
\text { Genetik, Universität Bielefeld }\end{array}$ \\
\hline RES167 $\Delta r e l$ & rel deletion mutant of RES167 & Wehmeier et al. (1998) \\
\hline KM6 & RES167 with integrated Tn5516invers, $\mathrm{Km}^{\mathrm{R}}$ & Tauch (1996) \\
\hline LW45 & RES167 carrying p $\triangle$ RPLK integrated in the chromsome & This work \\
\hline RES167 $\Delta r p l K$ & $r p l K$ deletion mutant of RES167 & This work \\
\hline \multicolumn{3}{|l|}{ E. coli strains } \\
\hline DH5 $\alpha \mathrm{MCR}$ & $\begin{array}{l}\mathrm{F}^{-} \text {supE44 endA thi-1 recA1 gyrA96 relA1 deoR } \Delta(\text { lac-argF }) \mathrm{U} 196 \phi 80 \mathrm{~d} \\
\text { lacZ M15 mcrA } \Delta(m m r \text { hsdRMS mcrBC) }\end{array}$ & Grant et al. (1990) \\
\hline S17-1 & $h s d R$ pro recA carrying RP4-2-Tc: : Mu in the chromosome & Simon et al. (1983) \\
\hline CF1648 & Wild-type MG1655 & Xiao et al. (1991) \\
\hline CF1652 & CF1648 arelA251 & Xiao et al. (1991) \\
\hline \multicolumn{3}{|l|}{ Plasmids } \\
\hline pKM6-6 & pK18mob carrying the Tn5516invers region of KM6 & Tauch (1996) \\
\hline pKM6-67 & HindIII deletion derivative of pKM6-6 & Tauch (1996) \\
\hline pK18mobsacB & E. coli mobilizable cloning vector containing a $s a c B$ derivative, $\mathrm{Km}^{\mathrm{R}}$ & Schäfer et al. (1994b) \\
\hline $\mathrm{p} \triangle \mathrm{RPLK}$ & $\begin{array}{l}\text { pK } 18 m o b s a c B \text { with a } 1.2 \mathrm{~kb} \text { PCR fragment comprising a } 12 \mathrm{bp} \\
\text { deletion derivative of the C. glutamicum rplK gene }\end{array}$ & This work \\
\hline pECM2 & E. coli-C.glutamicum shuttle vector, $\mathrm{Km}^{\mathrm{R}} \mathrm{Cm}^{\mathrm{R}}$ & Tauch et al. (1994) \\
\hline pECM3 & BamHI-BglII deletion derivative of pECM2, $\mathrm{Cm}^{\mathrm{R}}$ & This work \\
\hline pRPLK & pECM3 carrying a $1.2 \mathrm{~kb}$ PCR product with the wild-type $r p l K$ gene & This work \\
\hline
\end{tabular}

*ATCC, American Type Culture Collection; $\mathrm{Nx}^{\mathrm{R}}$, nalidixic acid resistance; $\mathrm{Km}^{\mathrm{R}}$, kanamycin resistance; Cm ${ }^{\mathrm{R}}$, chloramphenicol resistance.

by the neural network system PredictProtein (Rost et al., 1995).

Screening for spontaneous and induced thiostreptonresistant C. glutamicum mutants. For obtaining spontaneous thiostrepton-resistant colonies, different amounts of an exponentially growing culture $\left(10^{6}-10^{12}\right.$ cells) were spread on $\mathrm{LB}$ agar containing $1,2 \cdot 5,5$, or $10 \mu \mathrm{g}$ thiostrepton $\mathrm{ml}^{-1}$, respectively, and were incubated for $10 \mathrm{~d}$ at $30^{\circ} \mathrm{C}$. The screening for induced thiostrepton-resistant mutants was performed by chemical mutagenesis using ethyl methanesulfonate (EMS). An exponentially growing culture was concentrated 10-fold by centrifugation and resuspended in $0 \cdot 1 \mathrm{M}$ sodium phosphate buffer $\left(\mathrm{pH} \mathrm{7 \cdot 0)}\right.$ containing $1 \mathrm{mg}$ EMS $\mathrm{ml}^{-1}$ and incubated at $30{ }^{\circ} \mathrm{C}$ for $1 \mathrm{~h}$. Cells were then washed twice with LB medium and plated directly onto LB agar supplemented with different thiostrepton quantities (see above) and incubated at $30^{\circ} \mathrm{C}$.

Construction of a plasmid-encoded $r p / K$ deletion derivative. A $1.2 \mathrm{~kb}$ fragment comprising a $12 \mathrm{bp}$ deletion derivative of the $r p l K$ gene was amplified from the C. glutamicum chromosome using PCR with the oligonucleotides P1-up, P1-down, P2-up and P2-down (illustrated in Fig. 3). The PCR primers were deduced from the known nucleotide sequence (P1-up, nt 3291-3310; P1-down, nt 3916-3897; P2-up nt 3929-3948; P2down, nt 4534-4515) in such a way that P1-down and P2-up contained $10 \mathrm{bp} 5^{\prime}$ extensions which matched exactly the $5^{\prime}$ end of P2-up (nt 3929-3938) and P1-down (nt 3916-3907), respectively. First, two separate $600 \mathrm{bp}$ fragments were amplified from the target sequence. The first product, termed rplk-part1, was obtained with P1-up and P1-down and comprised the $5^{\prime}$ part of the $r p l K$ gene (nt 1-87) together with a $10 \mathrm{bp}$ extension corresponding to nt 100-109 of rplK, thereby carrying a $12 \mathrm{bp}$ gap within the amplified $r p l K$ portion compared to the used chromosomal template. The second fragment, named rplk-part2, was obtained with P2-up and P2down and consisted of the rplK 3' region (nt 78-435) carrying the same 12 bp gap ranging from nt 88-99 within the amplified $r p l K$ part. Accordingly, these two $r p l K$ PCR products shared a $20 \mathrm{bp}$ overlap region. When these intermediate products were mixed together, melted and reannealed, the top strand of rplk-part 1 could anneal to the bottom strand of rplk-part 2 in such a way that the two strands acted as primers on one another. Extension of this overlap and addition of the primers P1-up and P2-down created a $1.2 \mathrm{~kb}$ fragment which carried a $12 \mathrm{bp}$ deletion derivative of the $r p l K$ gene. The purified $1.2 \mathrm{~kb}$ PCR product was subsequently cloned into the $S m a I$-digested E. coli vector pK18mobsacB, yielding $\mathrm{p} \Delta \mathrm{RPLK}$. Therefore, $\mathrm{p} \Delta \mathrm{RPLK}$ comprised a mutant $r p l K$ gene, in which the $12 \mathrm{bp}$ deletion resulted in the loss of the tetrapeptide Pro-Ala-LeuGly in the corresponding L11 protein.

Measurement of (p)ppGpp accumulation. E. coli and C. glutamicum strains were screened for patterns of (p)ppGpp accumulation following the rapid screening method described previously (Cashel, 1994). Non-uniformly ${ }^{32} \mathrm{P}_{\mathrm{i}}$-labelled E. coli and C. glutamicum cells were obtained by growing the strains overnight on LB plates, followed by dispersion of single colonies in phosphate-free MOPS minimal medium and incubation with MOPS medium containing $10 \mu \mathrm{Ci}{ }^{32} \mathrm{P}_{\mathrm{i}} \mathrm{ml}^{-1}$ $\left(3.7 \times 10^{5} \mathrm{~Bq} \mathrm{ml}^{-1}\right)$ for $30 \mathrm{~min}$ at $37^{\circ} \mathrm{C}$. For amino acid starvation responses, the colonies were resuspended in aminoacid-free, phosphate-free MOPS medium supplemented with DL-serine hydroxamate $\left(1 \mathrm{mg} \mathrm{ml}^{-1}\right)$ and L-valine 
$\left(0 \cdot 5 \mathrm{mg} \mathrm{ml}^{-1}\right)$. The samples were mixed with $13 \mathrm{M}$ formic acid and three freeze-thaw cycles were performed. Afterwards, the samples were centrifuged and $5 \mu$ l samples of supernatants were subjected to one-dimensional polyethyleneimine (PEI) TLC developed with $1.5 \mathrm{M}$ potassium phosphate ( $\mathrm{pH} 3.4$ ). Nucleotide abundance was determined by autoradiography after overnight exposure of the PEI chromatograms at $-70^{\circ} \mathrm{C}$ to Cronex 4 films (Sterling). Analysis of the autoradiograms was performed with the help of a HP Scanjet $6100 \mathrm{C} / \mathrm{T}$ optical scanner (Hewlett Packard). The spot intensities were quantified densitometrically using the WinCam computer program (Cybertech). Accumulation data concerning the residual (p)ppGpp level are means of two independent PEI TLC assays.

Transcripitional assay. RNA of C. glutamicum was isolated by means of the Ribolyser instrument and the Hybaid Ribolyser RNA isolation kit. Blotting and hybridization experiments were performed as described by Sambrook et al. (1989). Hybridization signals were detected with the chemoluminescence substrate CSPD (Roche) and quantified densitometrically using the LabImage computer program (Labsoft). Gene probes of $r p l K$ and $r p l A$ were generated by PCR amplification using the Pwo DNA polymerase and the oligonucleotides rplK1 (nt 3758-3777), rplK2 (nt 4273-4292), rplA1 (nt 4490-4509) and rplA2 (nt 4976-4995), respectively. Subsequently, the PCR products were cloned into the vector pZEro-2 (Invitrogen). In vitro labelling of the cloned DNA fragments was performed with the DIG DNA labelling kit (SP6/T7) from Roche.

\section{RESULTS}

\section{The C. glutamicum rp/K gene encoding the large ribosomal subunit protein L11 is part of a DNA region also comprising secE, nusG and rpIA}

During analysis of transposon insertion sites in $C$. glutamicum, a $5.8 \mathrm{~kb}$ SalI DNA fragment was rescued from the chromosome of C. glutamicum KM6 (Tauch,
1996). The resulting plasmid, termed pKM6-6, carried the chromosomal $5^{\prime}$ - and $3^{\prime}$-flanking regions of the transposon insertion which are approximately $0.6 \mathrm{~kb}$ and $5 \cdot 2 \mathrm{~kb}$ in length (Fig. 1). Subsequently, pKM6-6 was cut with HindIII to construct the deletion derivative pKM6-67 (Tauch, 1996). DNA single-read sequence analysis of pKM6-67 using the universal sequencing primer (Fig. 1) revealed significant similarity to internal fragments of $r p l K$ genes from Gram-positive and Gramnegative bacteria (data not shown). Since the E. coli rplK gene is known to be involved in (p)ppGpp accumulation during amino acid starvation (Friesen et al., 1974), we decided to sequence the isolated $r p l K$ region.

The nucleotide sequence of a 5245 bp SalI-BglII chromosomal fragment present on pKM6-6 was determined by a primer walking strategy. The overall G $+\mathrm{C}$ content of the sequenced region is $52.1 \mathrm{~mol} \%$, which is in the range known from C. glutamicum chromosomal DNA (Malumbres et al., 1993). A computer-assisted analysis of the coding probability (Staden, 1986) revealed the presence of five ORFs with protein-coding character (Fig. 1). One of these ORFs, termed $r p l K$ (nt 3830-4267), encodes a protein of 145 amino acids with a calculated molecular mass of $15329 \mathrm{Da}$ and a deduced isoelectric point of 9.78. A potential ribosome-binding site (AAGGAA) is located 11 nucleotides upstream of the putative ATG start codon. Within the upstream region, neither E. coli consensus promoter sequences (Hawley \& McClure, 1983) nor any homology with conserved -35 and -10 regions of C. glutamicum (Patek et al., 1995 ) could be identified. Sequence comparison of the deduced RplK protein revealed significant similarity to ribosomal large-subunit proteins L11 (RplK) from other bacteria, including M. tuberculosis (EMBL Z92772), B. subtilis (EMBL Z99104), Streptomyces griseus (Küster

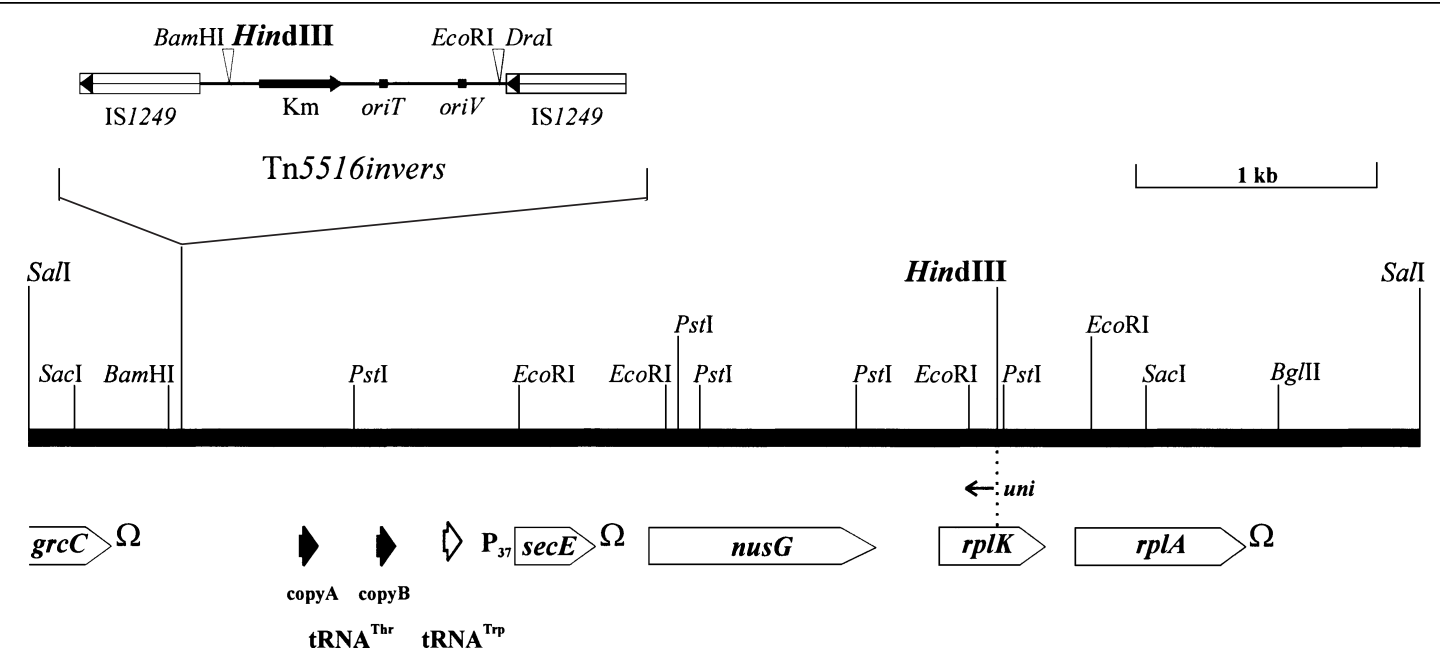

Fig. 1. Restriction map of the contiguous $5.8 \mathrm{~kb}$ rp/K region from $C$. glutamicum ATCC13032. The positions of the open reading frames ( $\operatorname{rrc} C$, secE, nusG, rp/K, rp/A) and the three tRNA genes (tRNA ${ }^{\text {Thr }}$ copyA, tRNA ${ }^{\text {Thr }}$ copyB, tRNA ${ }^{\text {Trp }}$ ) are indicated by arrows. The locations of the secE promoter fragment $\left(P_{37}\right.$; Patek et al., 1995) and the transcriptional terminators $(\Omega)$ of $\operatorname{grcC}$, secE, and downstream of $r p / A$ are marked. The insertion site of Tn5516invers, consisting of two IS1249 elements flanking pK18mob, is indicated. The HindIII restriction sites within Tn5516invers and the chromosomal fragment, used to construct a deletion derivative of the originally rescued plasmid, are typed in boldface. The position of the initial DNA single-read analysis with the universal sequencing primer (uni) is marked by an arrow. 
The C. glutamicum rplK gene region

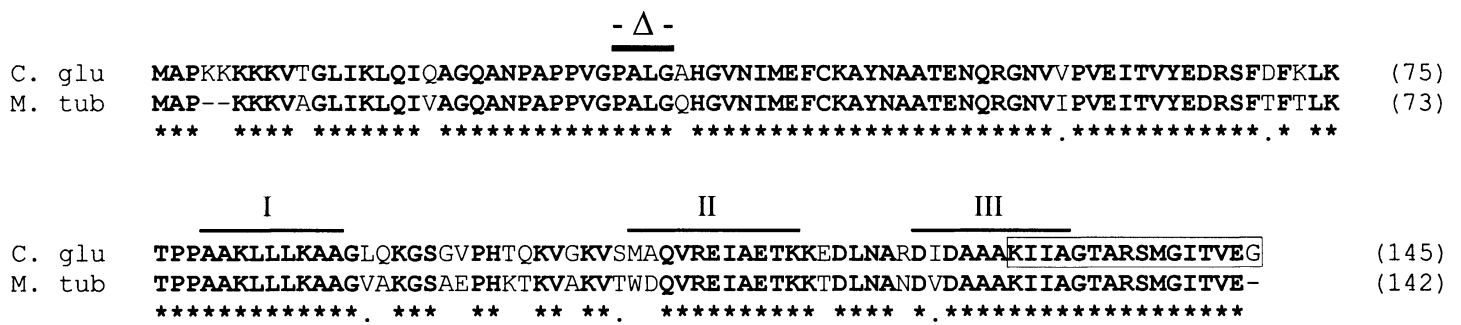

Fig. 2. Global amino acid sequence alignment of the deduced RplK protein from C. glutamicum (C. glu) with RplK from M. tuberculosis (EMBL Z92772) (M. tub). Identical amino acid residues are typed in bold and marked by asterisks; dots represent positions of conservative substitutions. Gaps $(-)$ were introduced by the ALIGN computer program to optimize the protein alignment. Numbers on the right correspond to amino acid positions relative to the start of each protein. The position of the tetrapeptide Pro-Ala-Leu-Gly, which is deleted in the C. glutamicum RplK mutant protein, is indicated (- $\Delta-$-). The C-terminal $\alpha$-helical domains I-III and the amino acid residues of the ribosomal protein L11 signature (boxed) are marked.

et al., 1998) and E. coli (EMBL U00006). The highest similarity was found to be with the M. tuberculosis RplK protein, sharing $85 \%$ amino acid identity and $88 \%$ similarity, by performing a global protein alignment (Fig. 2). PROSITE database searches with the deduced protein sequence identified a ribosomal protein L11 signature (PS00359) at the C-terminal end of the C. glutamicum RplK protein ranging from amino acid 130 to 145 . Furthermore, the neural network system PredictProtein (Rost et al., 1995) indicated three $\alpha$-helical structures within the $\mathrm{C}$-terminal half of the protein. This part of the RplK protein has been shown to exist in an extended and loosely folded conformation and is likely to be buried within the ribosomal structure in E. coli and Bacillus stearothermophilus (Choli, 1989; Xing \& Draper, 1996).

The downstream ORF, termed $r p l A$, revealed significant amino acid sequence similarity to the ribosomal largesubunit protein L1 (RplA) from a variety of microorganisms (Table 2). Two additional complete ORFs are located upstream of $r p l K$ (Fig. 1 and Table 2). The nus $G$ gene is likely to encode a transcriptional antiterminator protein. The protein deduced from the $\sec E$ gene displayed similarity to SecE proteins from several bacteria (Table 2). SecE proteins play an essential role in the bacterial protein translocation system.

\section{The wild-type $r p / K$ gene of $C$. glutamicum was replaced by an allele carrying a 12 bp in-frame deletion in a highly conserved $\mathrm{N}$-terminal region}

E. coli rplK mutants are unable to synthesize (p)ppGpp in response to amino acid starvation (Friesen et al., 1974). Several attempts to disrupt the $r p l K$ coding region of C. glutamicum by integration of a chloramphenicol resistance gene or to delete larger parts of the $r p l K$ gene failed (data not shown), indicating the essential nature of the RplK protein in this species. Therefore, we created a C. glutamicum mutant strain carrying a $12 \mathrm{bp}$ in-frame deletion within a highly conserved $\mathrm{N}$-terminal region of $r p l K$ which results in the loss of the tetrapeptide Pro-Ala-Leu-Gly from the L11 protein (Figs 2 and 3 ).
A DNA fragment comprising the rplK deletion derivative was obtained by a PCR strategy and was cloned into pK18mobsacB, yielding $\mathrm{p} \triangle \mathrm{RPLK}$. A detailed experimental procedure of this amplification is outlined in Methods and in Fig. 3. Subsequently, p $\Delta R P L K$ was transferred to E. coli S17-1 and integrated into the chromosome of C. glutamicum RES167 by conjugation resulting in C. glutamicum LW45. Excision of the plasmid can be selected for by growing the cells on LB agar containing $10 \%$ sucrose. Cells able to grow on this medium have lost the plasmid due to a second crossover event that either restores the wild-type gene arrangement or leads to a mutant strain carrying a defined $12 \mathrm{bp}$ deletion in the chromosome. However, all 97 clones tested contained the wild-type gene arrangement.

In a further approach, a DNA fragment comprising the wild-type $r p l K$ gene was obtained by PCR and was cloned into the SmaI-digested E. coli-C. glutamicum shuttle vector pECM3. The resulting plasmid, pRPLK, was subsequently transferred to C. glutamicum LW45 by conjugation. With the wild-type $r p l K$ copy provided in trans, we were able to select a second crossover and to replace the chromosomal $r p l K$ gene which was verified by sequencing of a chromosomal rplK PCR fragment originating from a sucrose-resistant clone (data not shown). The elimination of the plasmid-encoded $r p l K$ wild-type copy from the cell was subsequently achieved by heat-curing of pRPLK (Schäfer et al., 1994a). The complete loss of pRPLK was verified by Southern blot experiments using labelled pRPLK as a probe (data not shown). Finally, the resulting strain C. glutamiucm RES167 $\Delta r p l K$ carried a chromosomal 12 bp deletion within the $r p l K$ gene corresponding to the tetrapeptide Pro-Ala-Leu-Gly from the L11 protein.

\section{The deletion within the $r p / K$ gene leads to a tolerance of C. glutamicum against thiostrepton}

The RplK protein of E. coli forms part of the target site for the antibiotic thiostrepton, which binds the same domain of the 23S rRNA (Ryan et al., 1991). Therefore, mutations in the RplK protein render ribosomes thiostrepton resistant (Friesen et al., 1974). Since our former 
Table 2. Coding regions in the $C$. glutamicum rp/K gene region

\begin{tabular}{|lccccc|}
\hline Gene & $\begin{array}{c}\text { G+C } \\
\text { content } \\
(\mathbf{m o l} \%)\end{array}$ & $\begin{array}{c}\text { Length } \\
(\mathbf{a a})\end{array}$ & $\begin{array}{c}\text { Protein molecular mass } \\
(\mathbf{k D a})\end{array}$ & Identity (\%)/similarity (\%) & $\begin{array}{c}\text { GenBank } \\
\text { no. }\end{array}$ \\
\hline secE & $51 \cdot 8$ & 111 & $11 \cdot 5$ & $47 / 62$ to M. tuberculosis & AL123456 \\
nus $G$ & $53 \cdot 4$ & 318 & $34 \cdot 7$ & $38 / 50$ to $M$. tuberculosis & AL123456 \\
rplA & $57 \cdot 4$ & 236 & 25 & $71 / 84$ to M. tuberculosis & AL123456 \\
\hline
\end{tabular}
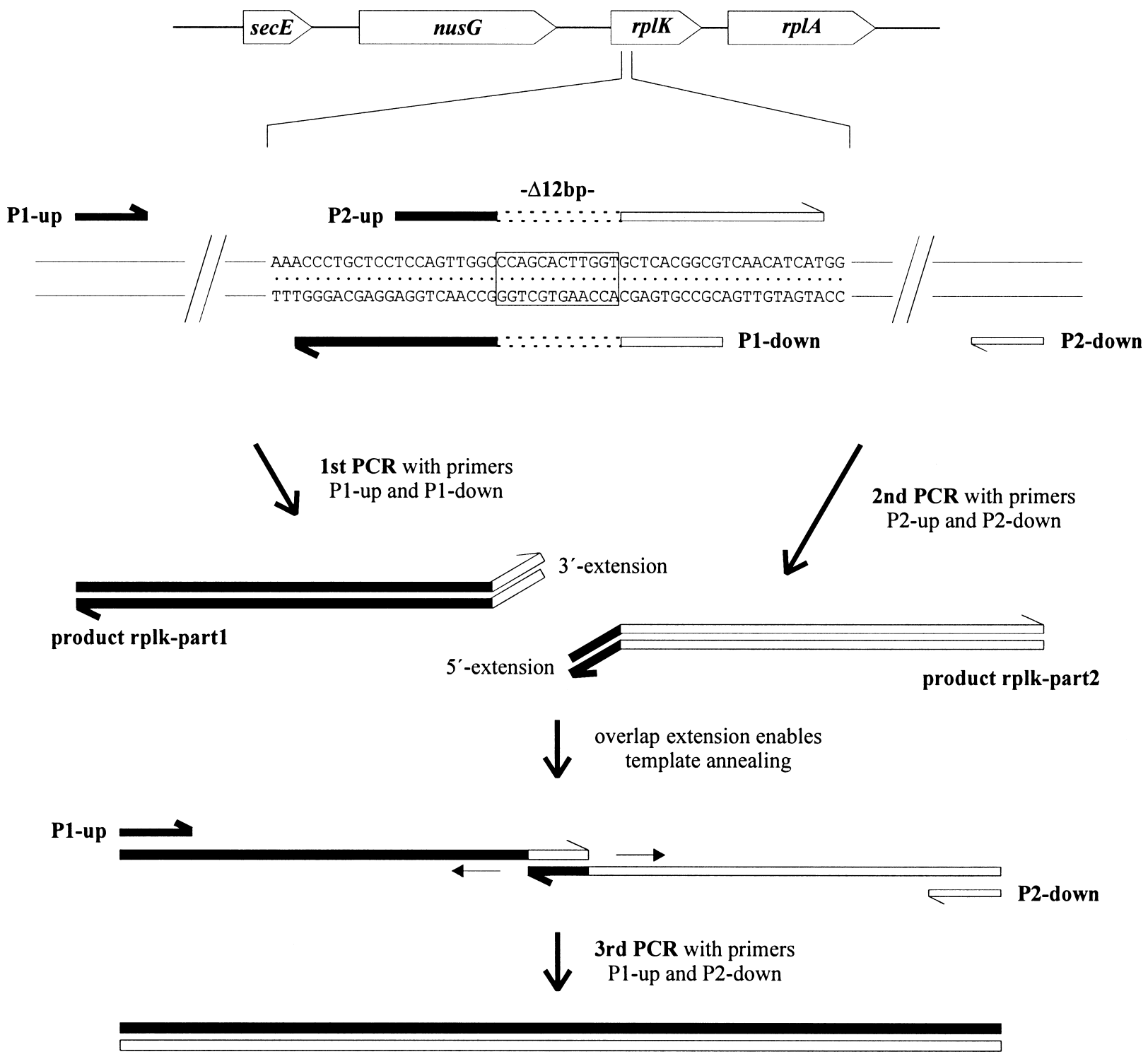

recombinant product rplk-del

Fig. 3. Strategy to obtain a $P C R$ fragment comprising a deletion derivative of the $C$. glutamicum rp/K gene. PCR with the primers P1-up, P1-down and P2-up, P2-down results in two products comprising the $5^{\prime}$ (rplk-part1) and 3' parts (rplkpart2) of the $C$. glutamicum rplK gene, respectively. Due to the $5^{\prime}$ extensions of P1-down and P2-up, both fragments contain the same gap within their respective $r p / K$ portion and share an overlap which enables annealing of the two intermediate products. In a further PCR reaction, extension of this overlap and the addition of primers P1-up and P2down create the recombinant product rplk-del, consisting of an rp/K deletion derivative (for further details see Methods). 


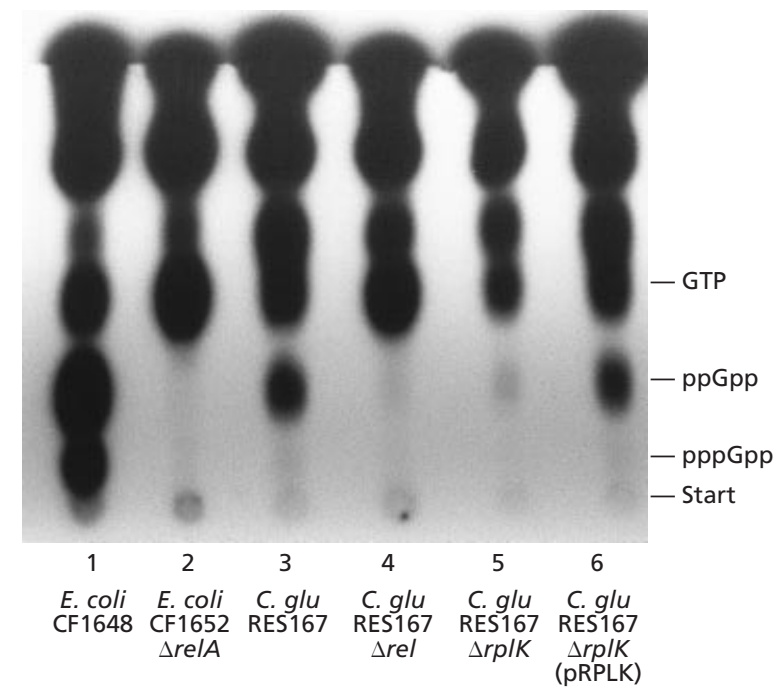

Fig. 4. (p)ppGpp accumulation of C. glutamicum wild-type and the $r p / K$ mutant in response to amino acid starvation. Lanes: 1, E. coli CF1648, wild-type; 2, CF1652, $\Delta$ relA; 3, C. glutamicum RES167, restriction-deficient wild-type derivative; 4, $\Delta$ rel derivative of RES167; $5, \Delta r p / K$ RES167 strain; $6, \Delta r p / K$ host of RES167, carrying plasmid pRPLK. Cultures were non-uniformly labelled with ${ }^{32} \mathrm{P}_{\mathrm{i}}$ and amino acid starved after growth on LB plates using DL-serine hydroxamate $\left(1 \mathrm{mg} \mathrm{ml}^{-1}\right)$ and L-valine $\left(0.5 \mathrm{mg} \mathrm{ml}^{-1}\right)$ as described in Methods. The positions of standards after PEI-TLC development in $1.5 \mathrm{M} \mathrm{KH}_{2} \mathrm{PO}_{4}$ are indicated at the right. C. glu, C. glutamicum.

attempts to isolate rplK mutants of C. glutamicum by screening for spontaneous or induced thiostreptonresistant colonies failed, we have analysed whether the constructed deletion within the rplK gene confers thiostrepton resistance in C. glutamicum. The growth of C. glutamicum RES167 was inhibited in the presence of $60 \mathrm{ng} \mathrm{ml}^{-1}$ thiostrepton in liquid growth medium expressed as the $50 \%$ value of the $\mathrm{OD}_{580}$, whereas the rplK mutant strain RES167 $\Delta r p l K$ grew in the presence of thiostrepton concentrations of up to $150 \mathrm{ng} \mathrm{m}^{-1}$. Thus, the C. glutamicum rplK mutant exhibited a $2 \cdot 5$ fold increased tolerance of thiostrepton.

\section{The C. glutamicum rp/K mutant is significantly impaired in (p)ppGpp accumulation upon amino acid starvation}

To assess the effect of the mutant $r p l K$ gene on (p)ppGpp accumulation, C. glutamicum RES167 $\Delta r p l K$ was subjected to amino acid starvation. Conditions of amino acid deprivation were induced by supplementation with $1 \mathrm{mg}$ DL-serine hydroxamate $\mathrm{ml}^{-1}$, which inhibits

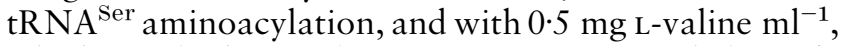
which resulted in isoleucine starvation (Cashel et al., 1996). Under such stress conditions, we detected (p)ppGpp in nucleotide extracts of C. glutamicum RES167 which co-migrates with (p)ppGpp synthesized by the E. coli wild-type (Fig. 4, lanes 1 and 3). This starvation response was completely abolished in E. coli CF1652 ( $\Delta$ relA) and C. glutamicum RES167 $\Delta$ rel, a
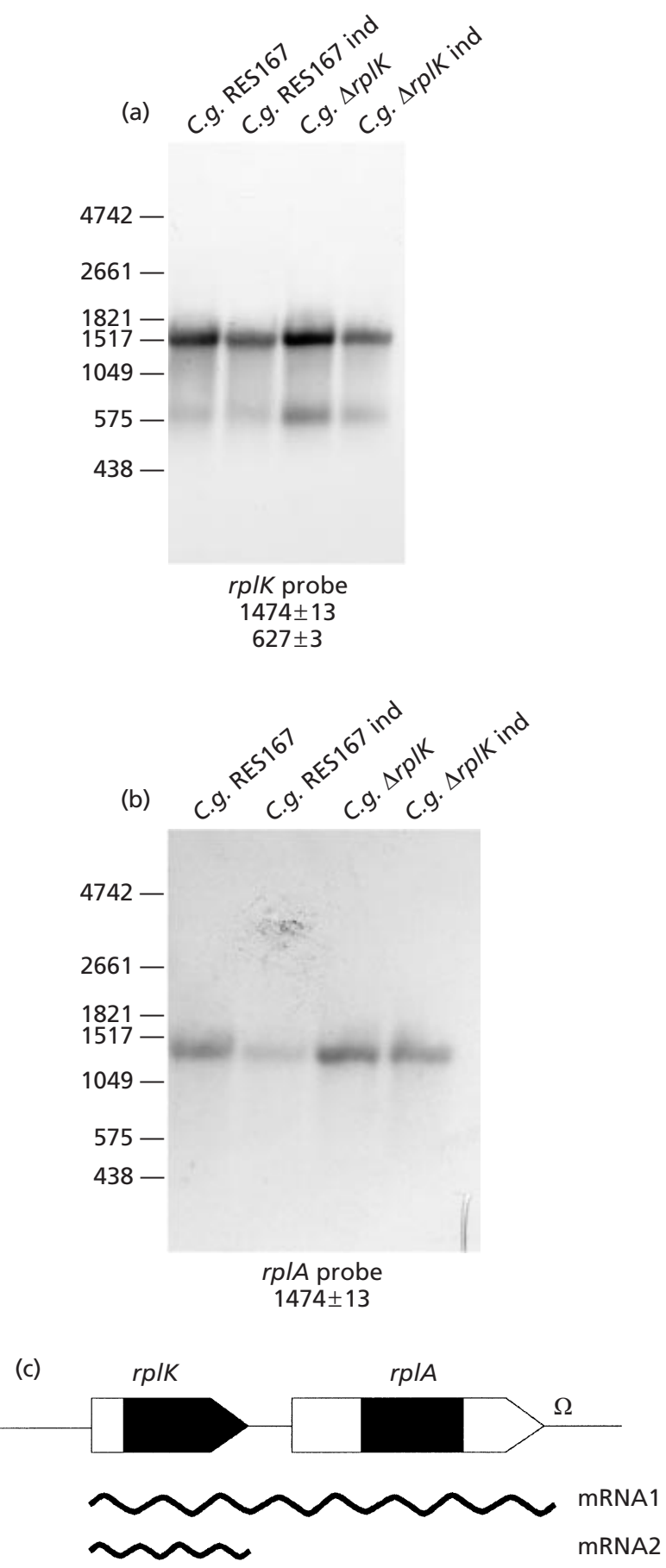

Fig. 5. Transcriptional analysis of the $r p / K-r p / A$ cluster. (a) Hybridization of RNA of C. glutamicum strains with an $r p / K$ probe. Amino acid starvation was induced (ind) in C. glutamicum RES167 and C. glutamicum RES167 $\Delta r p / K$ as described in Methods. Total RNA $(4 \mu \mathrm{g})$ was loaded in each lane. Size marker I (Roche) was used for length determination. The length of the transcripts was determined with the Lablmage software (Labsoft). (b) Hybridization of RNA of $C$. glutamicum strains with an rp/A probe. (c) Genetic organization of the gene cluster. The position of probes used for hybridization is marked. The two transcripts (mRNA1 and mRNA2) are shown by wavy lines. The presence of a putative transcriptional terminator $(\Omega)$ is indicated. C.g., C. glutamicum. 
mutant lacking a functional (p)ppGpp synthetase (Wehmeier et al., 1998) (Fig. 4, lanes 2 and 4). The C. glutamicum rplK mutant is significantly impaired in its ability to form (p)ppGpp following nutritional shiftdown (Fig. 4, lane 5). A computer-assisted analysis of the spot intensities, using the WinCam program, revealed only $14 \pm 5 \%$ (p)ppGpp compared to the (p)ppGpp level detected in the C. glutamicum wild-type. The (p)ppGpp accumulation assignment was verified by comparing the nucleotide activities of a limited series of sample dilutions (data not shown). The ability of $C$. glutamicum RES167 $\Delta$ rplK to produce (p)ppGpp could be completely restored by introducing a plasmidencoded $r p l K$ wild-type allele into the mutant strain (Fig. 4, lane 6), indicating that the $r p l K$ gene is required for (p)ppGpp accumulation upon amino acid deprivation.

\section{The rp/K and rplA genes of C. glutamicum form a bicistronic operon}

The results obtained by complementation analysis indicated that the $r p l K$ gene is involved in (p)ppGpp accumulation under amino acid starvation conditions. Nevertheless, another gene encoding a ribosomal protein $(r p l A)$ is located in close proximity to $r p l K$ (Fig. 1), suggesting a coordinate expression of these genes. In order to study the organization of the $r p l K$ and $r p l A$ genes of C. glutamicum, Northern blot hybridization with total RNA of RES167 and RES167 $\Delta r p l K$ and antisense RNA probes covering rplA and $r p l K$ was performed (Fig. 5). Two transcripts of $1.5 \mathrm{~kb}$ and $0.6 \mathrm{~kb}$ were observed on hybridization to the $r p l K$-specific probe (Fig. 5a). On hybridization to the rplA-specific probe only the $1.5 \mathrm{~kb}$ signal was detected (Fig. $5 \mathrm{~b}$ ). Considering the size of the $r p l K$ and $r p l A$ genes, these results indicate that a bicistronic mRNA covering $r p l K$ and $r p l A$ is formed and that additionally a monocistronic mRNA covering only $r p l K$ is synthesized. The length of the transcripts coincides well with the genetic organization of the $r p l K-r p l A$ cluster (Fig. 5c). The same transcriptional patterns of the $r p l K$ and $r p l A$ genes were observed in C. glutamicum RES167 and in the defined mutant strain C. glutamicum RES167 $\Delta$ rplK with the exception that the amount of their mRNAs slightly increased in RES167 $\Delta r p l K$ (Fig. 5).

\section{DISCUSSION}

In $E$. coli, the $r p l K$ gene, encoding the large ribosomal subunit protein L11, was shown to be indispensable for the activation of the RelA protein and consequently for synthesis of the stringent response mediator (p)ppGpp upon amino acid starvation (Friesen et al., 1974). A chromosomal region comprising the entire $r p l K$ gene of C. glutamicum was cloned during analysis of transposon insertions by a plasmid rescue technique. The deduced RplK protein showed strong similarity to all known ribosomal L11 proteins. In addition, the gene arrangement of the isolated region from C. glutamicum matched exactly the organization found in high and low $\mathrm{G}+\mathrm{C}$
Gram-positive bacteria including $M$. tuberculosis (EMBL Z92772), Str. coelicolor A3(2) (Puttikhunt et al., 1995), B. subtilis (EMBL Z99104), and Staphylococcus aureus (EMBL U96619). In all these organisms, $r p l K$ is preceded by two ORFs that are similar to nusG, encoding a transcription antiterminator protein, and secE, acting as a component of the protein export apparatus. The rplK gene is followed by an ORF homologous to rplA encoding the $50 \mathrm{~S}$ ribosomal protein L1. Moreover, the corresponding DNA regions in several Gram-negative bacteria, for example E. coli (EMBL U00006) and Helicobacter pylori (Tomb et al., 1997), also appear extensively conserved.

E. coli rplK mutants are strongly impaired in their ability to accumulate (p)ppGpp following a nutritional shift-down and exhibit, like relA mutants, a relaxed control of RNA synthesis. Therefore, the $r p l K$ locus was originally termed relC (Cashel et al., 1996). Almost identical results have been obtained in several other bacteria including B. subtilis (Smith et al., 1980), Bacillus megaterium (Stark \& Cundliffe, 1979), S. coelicolor A3(2) (Ochi et al., 1997), and S. griseus (Kawamoto et al., 1997), where mutations within the respective $r p l K /$ $\mathrm{relC}$ gene lead to an abolished stringent response. To determine the role of the C. glutamicum rplK gene in (p)ppGpp accumulation, we constructed and analysed an $r p l K$ mutant strain. All $r p l K$ mutants described so far have been isolated either by chemical mutagenesis or due to their property to confer thiostrepton resistance (Ochi, 1990). In contrast to these observations, thiostrepton-resistant C. glutamicum colonies could not be obtained under a variety of different screening procedures. Moreover, attempts to disrupt the $r p l K$ coding region, or to delete larger internal parts of $r p l K$ using the $s a c B$ system, which facilitates the detection of an allelic exchange by homologous recombination (Schäfer et al., 1994b) were unsuccessful, providing evidence that the RplK protein might be essential in $C$. glutamicum. Finally, the strain C. glutamicum RES167 $\Delta r p l K$ was constructed which carries a $12 \mathrm{bp}$ in-frame deletion within the $r p l K$ gene, resulting in the loss of the tetrapeptide Pro-Ala-Leu-Gly from the L11 protein. The deleted sequence represents the most highly conserved region of protein L11 in Gram-positive and Gramnegative bacteria and was shown to nearly prevent (p)ppGpp accumulation under amino acid starvation conditions in S. griseus (Kawamoto et al., 1997) and S. coelicolor A3(2) (Ochi et al., 1997).

(p)ppGpp accumulation assays in C. glutamicum wildtype and the $r p l K$ mutant strain established that the $r p l K$ gene is required for (p)ppGpp accumulation. Synthesis of (p)ppGpp invoked by amino acid starvation was almost abolished in the $r p l K$ mutants but not in the wildtype strain. The residual (p)ppGpp level corresponding to approximately $14 \pm 5 \%$ in the $r p l K$ mutant compared with the C. glutamicum wild-type agrees well with the (p)ppGpp accumulation level of about $10 \%$ remaining in rplK mutant strains of E. coli (Friesen et al., 1974), S. griseus (Kawamoto et al., 1997) and S. coelicolor A3(2) (Ochi et al., 1997). The deletion within the C. gluta- 
The C. glutamicum rplK gene region

micum rplK gene leads only to a $2 \cdot 5$-fold increase in the tolerance against thiostrepton, which is much lower than the 14-100-fold resistance known from the $r p l K$ mutant strains of $S$. griseus and S. coelicolor A3(2), respectively. However, the C. glutamicum wild-type is about 13-27-fold more sensitive to thiostrepton than both Streptomyces parental strains. Western blotting analysis revealed that the ribosomes from the $S$. griseus and $S$. coelicolor A3(2) rplK mutants incorporate the corresponding mutant L11 protein normally, although the level of incorporation is only approximately onethird to one-eighth of that observed with the wild-type L11 protein (Kawamoto et al., 1997; Ochi et al., 1997). Since C. glutamicum RES167 $\Delta r p l K$, like the $S$. griseus rplK mutant, lacks the highly conserved internal $12 \mathrm{bp}$ region, it seems likely that ribosomes from the $C$. glutamicum mutant strain also contain apparently lower amounts of mutant L11 protein, resulting in the strong impairment in (p)ppGpp accumulation. Despite the result of complementation in trans of the rplK mutant with the wild-type gene, we could not finally exclude the possibility that the defined $12 \mathrm{bp}$ in-frame deletion within $r p l K$ may cause a polar effect on $r p l A$ which itself may eventually contribute to the defect in (p)ppGpp accumulation. Northern analysis revealed that the $r p l K$ and rplA genes of C. glutamicum form a bicistronic operon since a transcript covering both genes was detected. However, the same transcription patterns with slightly higher levels of the $r p l K-r p l A$ transcript were observed in the defined $r p l K$ mutant strain, suggesting that effects on the stability of the messenger can be neglected.

Recently, we isolated a relA/spoT homologous gene from C. glutamicum, designated rel, which probably encodes a bifunctional enzyme with (p)ppGpp synthetase and (p)ppGppase activities. A rel mutant strain failed to synthesize (p)ppGpp in response to amino acid starvation (Wehmeier et al., 1998). In the present study, we established that a functional 50S ribosomal protein L11 in C. glutamicum is indispensable for (p)ppGpp accumulation upon nutritional deprivation. Therefore, it appears possible that the $C$. glutamicum Rel protein, like the homologues of E. coli (Cochran \& Byrne, 1974) and S. coelicolor A3(2) (Martinez-Costa et al., 1998), is ribosome-associated, requiring the RplK protein for activation of its (p)ppGpp synthetase function. With the molecular characterization of the rel and $r p l K$ genes from C. glutamicum we have identified two genes involved in (p)ppGpp synthesis in this industrially important micro-organism. Both genes are therefore involved in a global regulatory network response which might also influence amino acid production. This aspect is under current investigation in our laboratory.

\section{ACKNOWLEDGEMENTS}

We thank M. Cashel (Bethesda, USA) for kindly providing the E. coli strains CF1648 and CF1652. This work was supported by contract No. Bio-4-CT-960145 from the European Commission.

\section{REFERENCES}

Altschul, S. F., Madden, T. L., Schaeffer, A. A., Zhang, J., Zhang, J., Miller, W. \& Lipman, D. J. (1997). Gapped BLAST and PSI-BLAST: a new generation of protein database search programs. Nucleic Acids Res 25, 3389-3402.

Cashel, M. (1994). Detection of (p)ppGpp accumulation patterns in Escherichia coli mutants. In Methods in Molecular Genetics, vol. 3, Molecular Microbiology Techniques, Part A, pp. 341-356. Edited by K. W. Adolph. New York: Academic Press.

Cashel, M., Gentry, D. R., Hernandez, V. J. \& Vinella, D. (1996). The stringent response. In Escherichia coli and Salmonella: Cellular and Molecular Biology, 2nd edn, pp. 1458-1496. Edited by F. C. Neidhardt and others. Washington, DC: American Society for Microbiology.

Choli, T. (1989). Structural properties of ribosomal protein L11 from Escherichia coli. Biochem Int 19, 1323-1338.

Cochran, J. W. \& Byrne, R. W. (1974). Isolation and properties of a ribosome-bound factor required for $p p G p p$ and pppGpp synthesis in Escherichia coli. J Biol Chem 249, 353-360.

Cole, S. T., Brosch, R., Parkhill, J. \& 23 other authors (1998). Deciphering the biology of Mycobacterium tuberculosis from the complete genome sequence. Nature 393, 537-544.

Fraser, C. M., Gocayne, J. D., White, O. \& 7 other authors (1995). The minimal gene complement of Mycoplasma genitalium. Science 270, 397-403.

Friesen, J. D., Fiil, N. P., Parker, J. M. \& Haseltine, W. A. (1974). A new relaxed mutant of Escherichia coli with an altered $50 \mathrm{~S}$ ribosomal subunit. Proc Natl Acad Sci US A 71, 3465-3469.

Goldman, E. \& Jakubowski, H. (1990). Uncharged tRNA, protein synthesis, and the bacterial stringent response. Mol Microbiol 4, 2035-2040.

Grant, S. G. N., Jessee, J., Bloom, F. R. \& Hanahan, D. (1990). Differential plasmid rescue from transgenic mouse DNAs into Escherichia coli methylation-restriction mutants. Proc Natl Acad Sci U S A 87, 4645-4649.

Hawley, D. K. \& McClure, W. R. (1983). Compilation and analysis of Escherichia coli promotor sequences. Nucleic Acids Res 11, 2237-2255.

Kawamoto, S., Zhang, D. \& Ochi, K. (1997). Molecular analysis of the ribosomal L11 protein gene $(r p l K=$ relC) of Streptomyces griseus and identification of deletion allele. Mol Gen Genet 255, 549-560.

Kunst, F., Ogasawara, N., Moszer, I. \& 31 other authors (1997). The complete genome sequence of the gram-positive bacterium Bacillus subtilis. Nature 390, 249-256.

Küster, C., Piepersberg, W. \& Diestler, J. (1998). Cloning and transcriptional analysis of the $r p l K A$ - or $f 31-r p l J L$ gene cluster of Streptomyces griseus. Mol Gen Genet 257, 219-229.

Malumbres, M. \& Martin, J. F. (1996). Molecular control mechanisms of lysine and threonine biosynthesis in amino acidproducing corynebacteria: redirecting carbon flow. FEMS Microbiol Lett 143, 103-114.

Malumbres, M., Gil, J. A. \& Martin, J. F. (1993). Codon preferences in corynebacteria. Gene 134, 15-24.

Martinez-Costa, O. H., Arias, P., Romero, N. M., Parro, V., Mellado, R. P. \& Malpartida, F. (1998). The relA/spoT homologous gene from Streptomyces coelicolor encodes both ribosome-dependent (p)ppGpp-synthesizing and -degrading activities. J Bacteriol 180, 4123-4132.

Mechold, U., Cashel, M., Steiner, K., Gentry, D. \& Malke, H. 
(1996). Functional analysis of a relA/spoT gene homolog from Streptococcus equisimilis. J Bacteriol 178, 1401-1411.

Metzger, S., Sarubbi, E., Glaser, G. \& Cashel, M. (1989). Protein sequences encoded by the relA and spoT genes of Escherichia coli are interrelated. J Biol Chem 264, 9122-9125.

Murray, K. D. \& Bremer, H. (1996). Control of spoT-dependent ppGpp synthesis and degradation in Escherichia coli. J Mol Biol 259, 41-57.

Myers, E. W. \& Miller, W. (1988). Optimal alignments in linear space. Comput Appl Biosci 4, 11-17.

Ochi, K. (1990). Streptomyces relC mutants with an altered ribosomal protein ST-11 and genetic analysis of a Streptomyces griseus relC mutant. J Bacteriol 172, 4008-4016.

Ochi, K., Zhang, D., Kawamoto, S. \& Hesketh, A. (1997). Molecular and functional analysis of the ribosomal L11 and S12 protein genes ( $r p l K$ and $r p s L$ ) of Streptomyces coelicolor A3(2). Mol Gen Genet 256, 488-498.

Patek, M., Eikmanns, B. J., Patek, J. \& Sahm, H. (1995). Promotors from Corynebacterium glutamicum: cloning, molecular analysis and search for a consensus motif. Microbiology 142, 1297-1309.

Puttikhunt, C., Nihira, T. \& Yamada, Y. (1995). Cloning, nucleotide sequence, and transcriptional analysis of the nus $G$ gene of Streptomyces coelicolor A3(2), which encodes a putative transcriptional antiterminator. Mol Gen Genet 247, 118-122.

Rost, B., Casadio, R., Fariselli, P. \& Sander, C. (1995). Prediction of helical transmembrane segments at $95 \%$ accuracy. Protein Sci 4 , 521-533.

Ruklisha, M., Viesturs, U. \& Labane, L. (1995). Growth control and ppGpp synthesis in Brevibacterium flavum cells at various medium mixing rates and aeration intensities. Acta Biotechnol 15, 41-48.

Ryals, J., Little, R. \& Bremer, H. (1982). Control of rRNA and tRNA synthesis in Escherichia coli by guanosine tetraphosphate. J Bacteriol 151, 1261-1268.

Ryan, P. C., Lu, M. \& Draper, D. E. (1991). Recognition of the highly conserved GTPase center of $23 \mathrm{~S}$ ribosomal RNA by ribosomal protein $\mathrm{L} 11$ and the antibiotic thiostrepton. J Mol Biol 221, 1257-1268.

Sambrook, J., Fritsch, E. F. \& Maniatis, T. (1989). Molecular Cloning: a Laboratory Manual, 2nd edn. Cold Spring Harbor, NY: Cold Spring Harbor Laboratory.

Schäfer, A., Kalinowski, J., Simon, R., Seep-Feldhaus, A.-H. \& Pühler, A. (1990). High-frequency conjugal plasmid transfer from Gram-negative Escherichia coli to various Gram-positive coryneform bacteria. J Bacteriol 172, 1663-1666.

Schäfer, A., Schwarzer, A., Kalinowski, J. \& Pühler, A. (1994a). Cloning and characterization of a DNA region encoding a stress- sensitive restriction system from Corynebacterium glutamicum ATCC13032 and analysis of its role in intergenic conjugation with Escherichia coli. J Bacteriol 176, 7309-7319.

Schäfer, A., Tauch, A., Jäger, W., Kalinowski, J., Thierbach, G. \& Pühler, A. (1994b). Small mobilizable multi-purpose cloning vectors derived from Escherichia coli plasmids pK18 and pK19: selection of defined deletions in the chromosome of Corynebacterium glutamicum. Gene 145, 69-73.

Simon, R., Priefer, U. \& Pühler, A. (1983). A broad host range mobilization system for in vivo genetic engineering: transposon mutagenesis in Gram-negative bacteria. Bio/Technology 1, 784-794.

Smith, I., Paress, P., Cabane, K. \& Dubnau, E. (1980). Genetics and physiology of the rel system of Bacillus subtilis. Mol Gen Genet 178, 271-279.

Staden, R. (1986). The current status and portability of our sequence handling software. Nucleic Acids Res 14, 217-232.

Stark, M. \& Cundliffe, E. (1979). Requirement for ribosomal protein BM-L11 in stringent control of RNA synthesis in Bacillus megaterium. Eur J Biochem 102, 101-105.

Tauch, A. (1996). Entwicklung von Transposonmutagenesesystemen für Aminosäure-produzierende Corynebakterien. $\mathrm{PhD}$ thesis, University of Bielefeld.

Tauch, A., Kirchner, O., Wehmeier, L., Kalinowski, J. \& Pühler, A. (1994). Corynebacterium glutamicum DNA is subjected to methylation-restriction in Escherichia coli. FEMS Microbiol Lett 123, 343-347.

Tauch, A., Kassing, F., Kalinowski, J. \& Pühler, A. (1995). The Corynebacterium xerosis composite transposon Tn5432 consists of two identical insertion sequences, designated IS1249, flanking the erythromycin resistance gene erm CX. Plasmid 34, 119-131.

Tomb, J. F., White, O., Kerlavage, A. R. \& 39 other authors (1997). The complete genome sequence of the gastric pathogen Helicobacter pylori. Nature 388, 539-547.

Wehmeier, L., Schäfer, A., Burkovski, A., Krämer, R., Mechold, U., Malke, H., Pühler, A. \& Kalinowski, J. (1998). The role of the Corynebacterium glutamicum rel gene in (p)ppGpp metabolism. Microbiology 144, 1853-1862.

Xiao, H., Kalman, M., Ikehara, K., Zemel, Z., Glaser, G. \& Cashel, M. (1991). Residual guanosine 3',5'-bispyrophosphate synthetic activity of relA null mutants can be eliminated by spoT null mutations. J Biol Chem 266, 5980-5990.

Xing, Y. \& Draper, D. E. (1996). Cooperative interaction of RNA and thiostrepton antibiotic with two domains of ribosomal protein L11. Biochemistry 35, 1581-1588.

Received 26 October 2000; accepted 20 November 2000. 